\title{
Sequential design and global optimization of local power system stabilizer and wide-area HVDC stabilizing controller
}

\author{
Yong $\mathrm{LI}^{1}$, Fang $\mathrm{LIU}^{2}$, Yijia $\mathrm{CAO}^{1}$
}

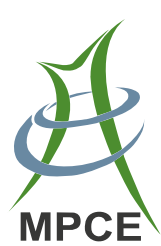

\begin{abstract}
A sequential design and global optimization method is proposed to coordinately design local and widearea controllers to enhance the overall stability of largescale power system. The sequential design is used to assign the distributed local power system stabilizer (LPSS) and high-voltage direct current (HVDC) wide-area stabilizing controller (HVDC-WASC) to the concerned damping modes. The global optimization is used to simultaneously optimize all the overall control gains of LPSSs and HVDCWASC. Moreover, the optimization model, which has an adaptive ability of searching and updating dominant oscillation modes, is established. Both the linear analysis and nonlinear simulation results verify the effectiveness of the proposed design method in enhancing the stability of large-scale power systems.
\end{abstract}

Keywords Local control, Wide-area control (WAC), Local power system stabilizer (LPSS), High-voltage direct current (HVDC), Wide-area stabilizing control (WASC)

CrossCheck date: 15 April 2015

Received: 16 July 2014/ Accepted: 16 April 2015/Published online: 15 August 2015

(C) The Author(s) 2015. This article is published with open access at Springerlink.com

$\triangle$ Yong LI

yonglichi@gmail.com

Fang LIU

csuleon@gmail.com

Yijia CAO

yjcao@hnu.edu.cn

1 College of Electrical and Information Engineering, Hunan University, Changsha 410082, China

2 School of Information Science and Engineering, Central South University, Changsha 410083, China

\section{Introduction}

More sensors, more communication, more computation, and more control are the general characteristics of the future smart transmission grid [1]. Following this trend, the wide use of synchronized phasor measurement technology enables the application of wide-area control (WAC) [1-3]. With the development of the phasor measurement unit (PMU) based wide-area measurement system (WAMS), it becomes convenient to measure, gather, and process large amounts of data within global range. Accordingly, the WAC strategies [1, 4-8], such as the regional voltage control, small signal stability control, and frequency control can be implemented to improve the overall stability of the smart transmission grid.

Although WAC strategy can improve the stability of power systems, it is used for part of stability problems, and the local control should still be involved to coordinate the WAC for the overall stability enhancement. Taking the small signal stability as an example, high-voltage direct current (HVDC) supplementary wide-area stabilizing control (WASC) is used to dampen inter-area oscillations, and the local power system stabilizer (LPSS) is used for local oscillations [9-11]. The interaction and coordination between LPSS and HVDC-WASC should be considered carefully. In practice, there are numerous LPSSs at the generator sides. Therefore, it is important for the simultaneous coordination of LPSSs and HVDC-WASC.

Up to now, several simultaneous tuning methods have been proposed for the optimization of power system damping controllers [12-16], which are mainly featured as: (1) focus on the local damping controllers, while the interactions between local and WACs are not considered; (2) these methods need to simultaneously optimize the control gain and the phase compensation blocks. Taking the 
16-generator 5-area system as an example [17], there are three variables (one control gain and at least two lead-lag time constants) for one LPSS. For the whole system, at least $16 \times 3=48$ optimization variables should be considered simultaneously. Therefore, it has to take more time to optimize theses parameters, which is uneconomic; (3) these methods achieve the overall stability enhancement via the coordination control of all LPSSs. Therefore, sometimes, if one or two LPSSs miss the control function, it may impact on other LPSSs and even destroy the overall stability; (4) the objective functions of these methods focus on all the oscillation modes including the stable modes, which inevitably increases the computational cost.

In this paper, a sequential design and global optimization method is proposed to coordinate LPSSs and HVDCWASC. This method focuses on the improvement of all the dominant oscillation modes and the overall stability of power systems. In this method, sequential design is used for the phase compensation design of these local and widearea stabilizing controllers, and global optimization is used to further optimize the overall control gains.

The structure of this paper is organized as follows. Following this part, section 2 presents the guideline of sequential deign, and the controller structure; section 3 presents the objective function and commutating algorithm of the global optimization; a detailed case study, which includes linear analysis and nonlinear simulation, is given in section 4, and the conclusion is addressed in section 5 .

\section{Sequential design}

\subsection{Structure of LPSS and HVDC-WASC}

Figure 1 shows the controller structures of LPSS and HVDC-WASC. The control principle of LPSS is to produce a component of electrical torque in phase with the rotor speed deviations via the auxiliary control on the excitation system [18]. It consists of one control gain, one washout block, and two phase compensation blocks, as shown in Fig. 1a. For HVDC-WASC, it also adopts the same controller structure. However, unlike LPSS, the control input of the HVDC-WASC is selected from widearea signals, in purpose of achieving effective damping performance on low-frequency oscillations.

Moreover, both the HVDC converter pole-controllers at the rectifier and the inverter side of HVDC transmission system can be selected to introduce the supplementary wide-area stabilizing signal. But considering that at the rectifier side, it has the simpler control strategy and controller structure, thus, the HVDC-WASC is implemented at the rectifier side.

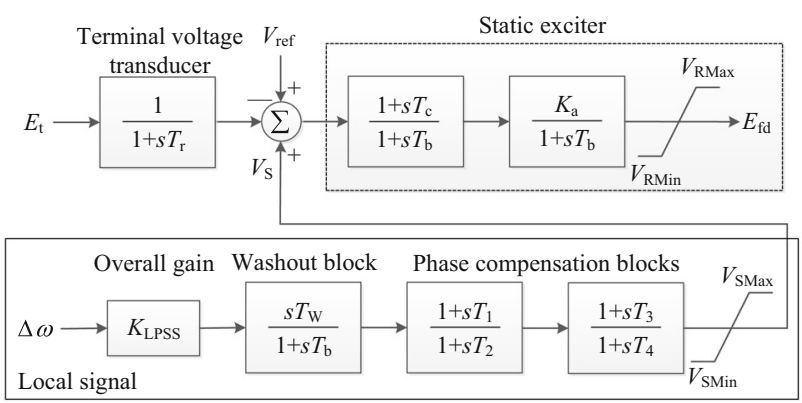

(a) LPSS

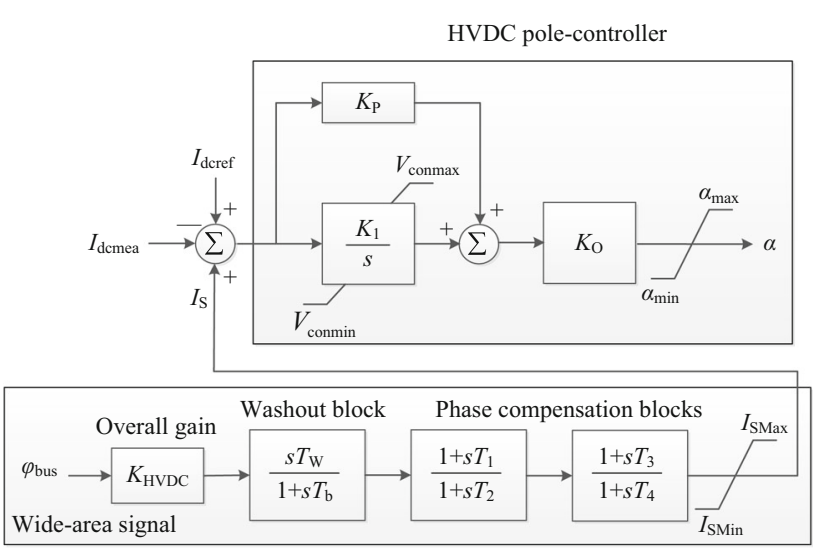

(b) HVDC-WASC

Fig. 1 Controller structures of LPSS and HVDC-WASC

\subsection{Design guideline}

The objective of the sequential design is to assign each LPSS and HVDC-WASC to play an effective damping ability at the around of the concerned oscillation frequency, by means of determining the phase compensation blocks one by one. To reach this objective, the practical design methods, i.e., the phase matching [17] and the phase compensation [18] methods, are applied to design the LPSS and the HVDC-WASC, respectively. In addition, for each step, the root locus analysis is applied to check the effectiveness of the designed phase compensation blocks. The guideline for the sequential deign is planned as follows:

1) For HVDC-WASC, initially design the lead-lag blocks for phase compensation at the around of the inter-area oscillation frequency $(0.2 \mathrm{~Hz} \leq f \leq 0.8 \mathrm{~Hz})$, and check the compensation performance by means of the root locus analysis. If an effective performance cannot be obtained, keep adjust the lead-lag time constants.

2) For LPSSs assigned for local modes $(f>1.0 \mathrm{~Hz})$, in each step, initially design the lead-lag blocks to match the phase lag between the exciter voltage reference and the generator electric power output. Then, check the matching performance by means of the root locus 
analysis. If an effective performance can be obtained, continue checking the effective performance on other oscillation modes, and then determine these effective dominant damping modes. On the contrary, adjust the lead-lag time constants again until such effectiveness achieves, and then re-check other oscillation modes.

3) For LPSSs assigned to act on the other LFO modes $(0.8 \mathrm{~Hz}<f<2.0 \mathrm{~Hz})$, in each step, firstly, check the designed LPSSs in 2) on the LFO modes that will be concerned in 3). If these LPSSs cannot be in an efficient damping performance, the lead-lag blocks of the LPSS should be linked to the generator with the highest participation factor, then the matching performance should be checked by means of the root locus analysis. On the contrary, if these LPSSs can get effective damping, the LPSS linked to the generator with the second highest participation factor can be considered.

\section{Global optimization}

\subsection{Objective function}

A global optimization method is further proposed to search out optimal control gains for LPSSs and HVDCWASC. The optimization object is to globally reconfigure the eigenvalues and ensure all the LFO modes from weak damping ratios $(\rho<0.05)$ to strong damping ratio ( $\rho \geq 0.05$ ). The objective function is established as

$\min . F=\left\{\begin{array}{l}\text { if existing, } \rho_{i}<0.05, \min \left(\sum_{i=1}^{n}\left(1-\rho_{i}\right)\right)_{j} \\ \text { else, } 0.95\end{array}\right.$

subject to $0 \leq K_{\mathrm{LPSS}, \text { HVDC-WASC }} \leq 100$

where $F$ is the function value whose final value should be $0.95 ; \rho_{i}$ is the damping ratio of the $i^{\text {th }}$ oscillation mode, which is calculated in every iterative step; $n$ is the number of the weak damping ratios; $j$ is the number of the operating conditions; and $K$ is the overall gain of each controller.

Unlike other optimization methods [12-16], the number of the weak oscillation modes during the iteration is decreased step by step. Therefore, the proposed method has an adaptive ability that can reduce the computational cost and accelerate the convergence.

\subsection{Optimization algorithm}

Figure 2 shows the flowchart of the global optimization method. The pattern search algorithm is applied to solve (1), which includes three parts.
1) Stability assessment. Stability assessment is initially applied to find all the weak oscillation modes with the damping ratios $(\rho<0.05)$ for objective function (1) After the output of the initial optimized control gains, the stability assessment is re-implemented to identify whether the unstable oscillation modes $(\rho<0.05)$ exist or not.

2) Optimization iteration. The iteration is based on the pattern search algorithm, during which, the former generated control gains are used to setup the later closed-loop system. After stability assessment, a new (1) is formed for next-step implementation of the pattern search algorithm.

3) Global iteration. This is the complementarity of the optimization iteration. The optimization iteration may sometimes falls into an infinite loop. In such a case, the the initial overall control gains should be resetted, and the global iteration can be implemented to restart the optimization iteration.

\section{Case study}

The 16-machine test system is used to illustrate the proposed method, as shown in Fig. 3. To improve the interconnected ability, an HVDC system is configured

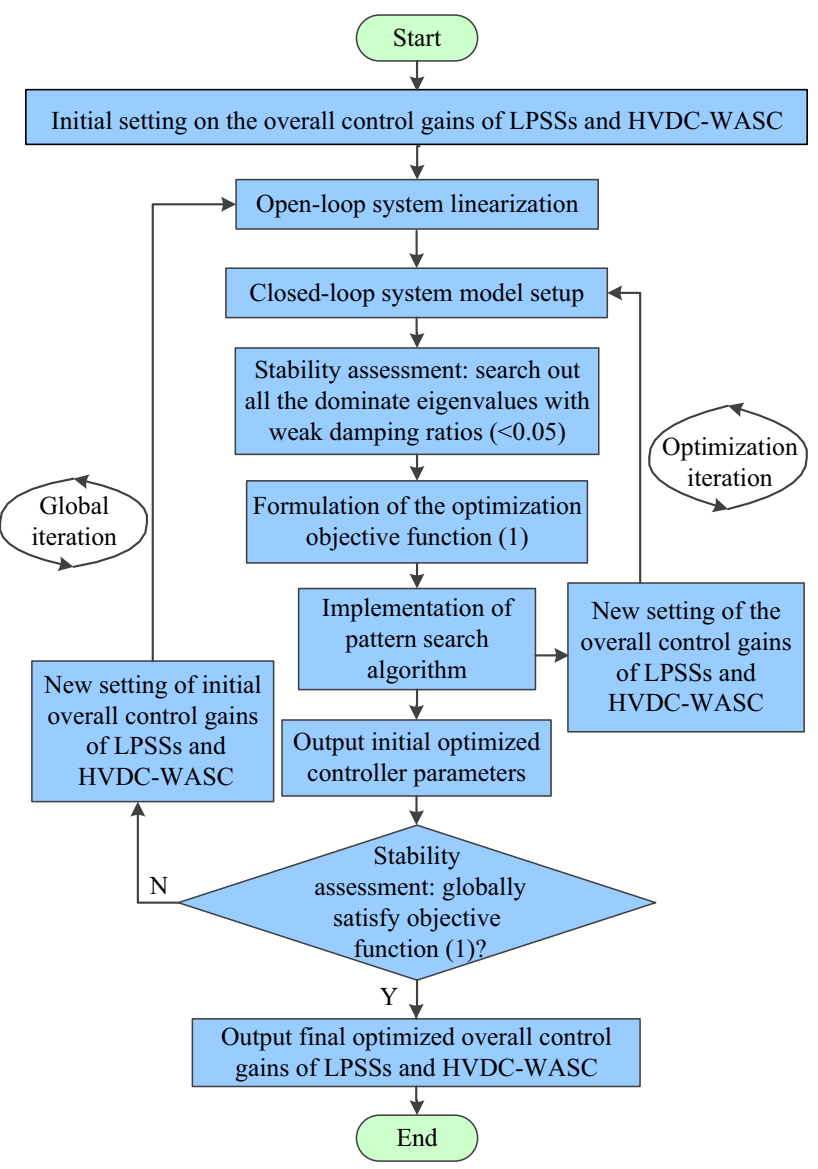

Fig. 2 Flowchart of pattern search algorithm based global optimization 
between Bus-52 (in Area-3) and 51 (in Area-4). The detailed system description including network data and dynamic data for the generators and the excitation systems can be found in [17].

\subsection{Design results}

Table 1 shows the designed results on LPSSs and HVDC-WASC. It can be seen that after 15 steps, the

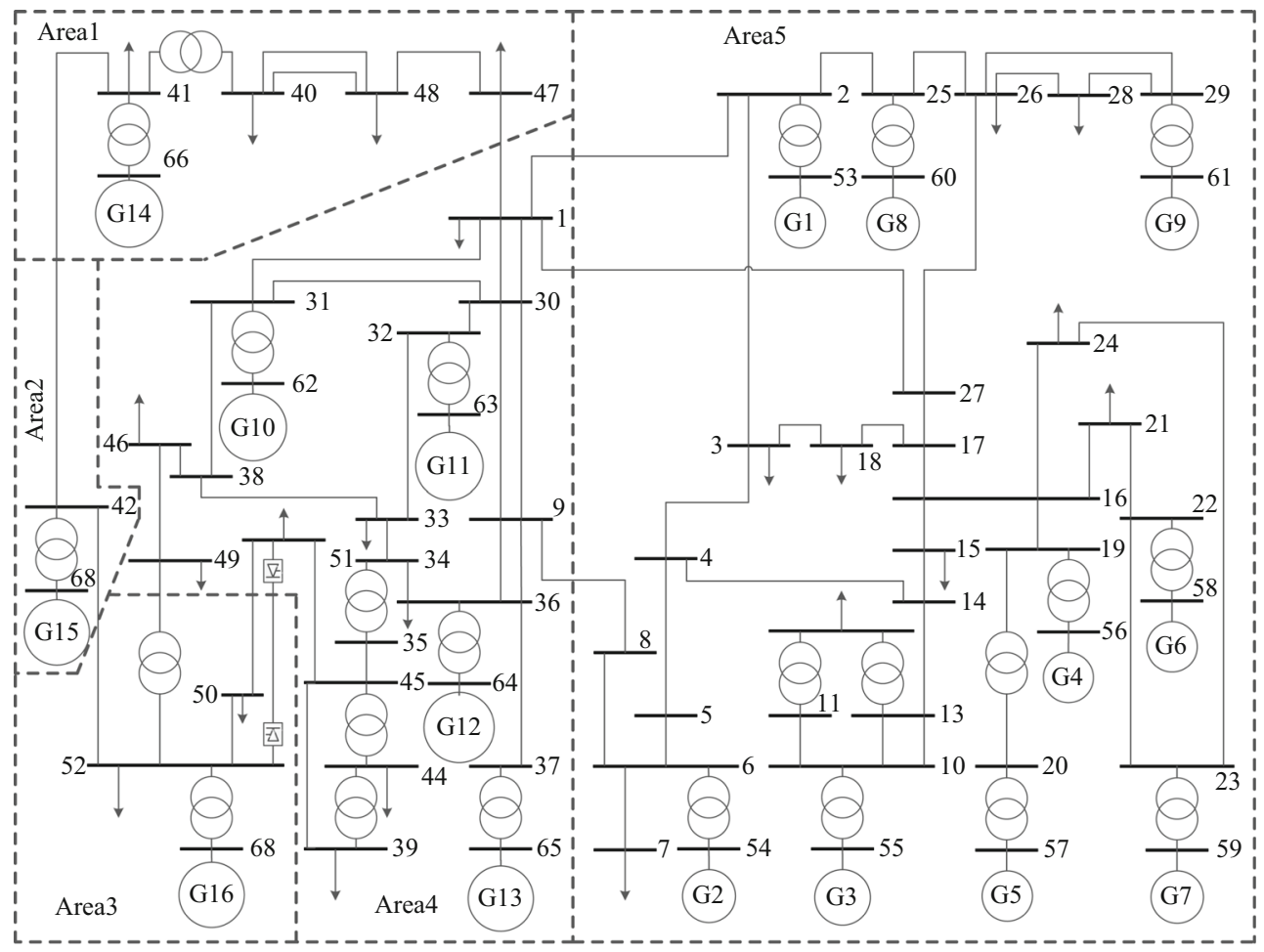

Fig. 3 Modified 16-machine 68-bus test system

Table 1 Results of sequential design and global optimization for LPSSs and HVDC-WASC

\begin{tabular}{|c|c|c|c|c|c|c|c|}
\hline \multirow[t]{2}{*}{ Step } & \multirow[t]{2}{*}{ Type } & \multicolumn{5}{|c|}{ Sequential design results } & \multirow{2}{*}{$\begin{array}{l}\text { Global optimization } \\
\text { results } \\
K\end{array}$} \\
\hline & & $T_{\mathrm{w}}$ & $T_{1}$ & $T_{2}$ & $T_{3}$ & $T_{4}$ & \\
\hline 1 & G16-LPSS & 10 & 0.03 & 0.02 & 0.03 & 0.02 & 94.0 \\
\hline 2 & G13-LPSS & 10 & 0.05 & 0.01 & 0.04 & 0.01 & 93.0 \\
\hline 3 & G14-LPSS & 10 & 0.04 & 0.02 & 0.04 & 0.03 & 94.0 \\
\hline 4 & G12-LPSS & 10 & 0.11 & 0.01 & 0.10 & 0.01 & 98.0 \\
\hline 5 & G2-LPSS & 10 & 0.10 & 0.02 & 0.10 & 0.02 & 92.5 \\
\hline 6 & G9-LPSS & 10 & 0.06 & 0.02 & 0.06 & 0.02 & 98.0 \\
\hline 7 & G5-LPSS & 10 & 0.08 & 0.02 & 0.08 & 0.02 & 93.0 \\
\hline 8 & G1-LPSS & 10 & 0.11 & 0.02 & 0.12 & 0.02 & 5.0 \\
\hline 9 & G3-LPSS & 10 & 0.09 & 0.02 & 0.08 & 0.02 & 93.0 \\
\hline 10 & G10-LPSS & 10 & 0.10 & 0.02 & 0.09 & 0.02 & 93.0 \\
\hline 11 & G4-LPSS & 10 & 0.10 & 0.02 & 0.10 & 0.02 & 5.0 \\
\hline 12 & G7-LPSS & 10 & 0.09 & 0.02 & 0.08 & 0.02 & 20.0 \\
\hline 13 & G8-LPSS & 10 & 0.09 & 0.01 & 0.09 & 0.01 & 100.0 \\
\hline 14 & G11-LPSS & 10 & 0.08 & 0.02 & 0.05 & 0.02 & 93.0 \\
\hline 15 & HVDC-WASC & 10 & 0.05 & 0.01 & 0.05 & 0.01 & 3.0 \\
\hline
\end{tabular}


sequential design proposed in Sect. 3 can be accomplished, and the lead-lag time constants of all controllers can be obtained. After this, all the control gains are optimized by means of the global optimization proposed in Sect. 4.

\subsection{Design results}

The eigenvalue analysis is used to validate the proposed method. Fig. 4 shows the dominant eigenvalues of the system with or without optimized controllers. It is clear that after optimization, all the dominant eigenvalues are placed into the prospective region where the damping ratio is more than $0.05(\rho \geq 0.05)$, thus all the LFO oscillation

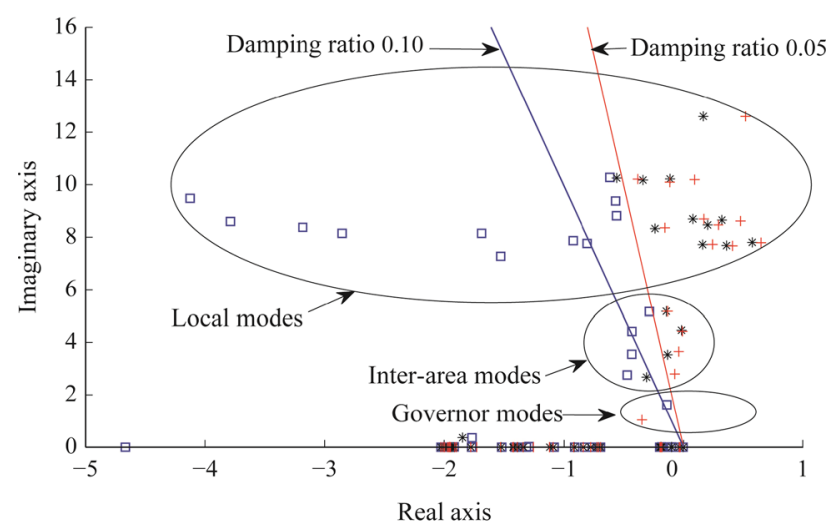

$+, *, \square$ Dominant eigenvalues of the power system without, with the initial, and with the optimized LPSSs and HVDC-WASC

Fig. 4 Optimization results modes are effectively damped. Table 2 further gives the detailed results. It can be seen that all the damping ratios are increased when the optimized controllers are used in the system.

Furthermore, in order to validate the control performance under different operating conditions, two typical operating conditions are considered: (1) two tie-lines between Area 4 and 5 are out of service; (2) $+20 \%$ loading increase on all the buses with P,Q loads. Fig. 4 shows the dominant eigenvalues on the system with these different operating conditions. It can be seen that all the eigenvalues are placed at the acceptance region. Moreover, the results also reflect the robustness of the optimized LPSSs and

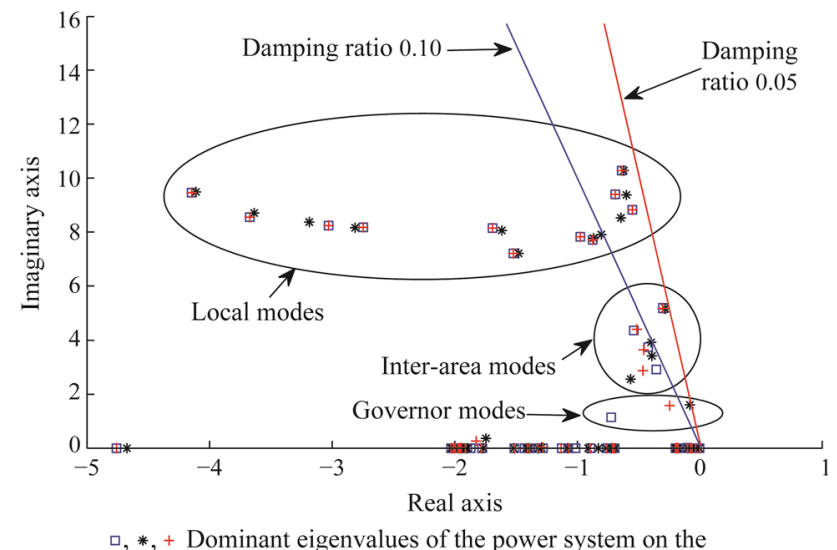

$\square, *,+$ Dominant eigenvalues of the power system on the normal operating condition, on the operating condition-1, and -2

Fig. 5 Impacts of different operating conditions on the test system with the optimized LPSSs and HVDC-WASC

Table 2 Dominant eigenvalues of the test system with or without LPSSs and HVDC-WASA

\begin{tabular}{|c|c|c|c|c|}
\hline \multirow[t]{2}{*}{ Oscillation mode } & \multicolumn{2}{|c|}{ With initial LPSSs and HVDC-WASC } & \multicolumn{2}{|c|}{ With optimized LPSSs and HVDC-WASC } \\
\hline & Dominant eigenvalues & Damping ratio & Dominant eigenvalues & Damping ratio \\
\hline Exciter mode & $0.3075 \pm 1.8310 i$ & -0.1656 & $-0.1397 \pm 1.6193 i$ & 0.086 \\
\hline \multirow[t]{4}{*}{ Inter-area mode mode } & $-0.3089 \pm 2.6691 i$ & 0.1149 & $-0.4705 \pm 2.7586 i$ & 0.1681 \\
\hline & $-0.1338 \pm 3.5230 i$ & 0.038 & $-0.4327 \pm 3.5487 i$ & 0.121 \\
\hline & $-0.0177 \pm 4.4579 i$ & 0.004 & $-0.4314 \pm 4.4132 i$ & 0.0973 \\
\hline & $-0.1447 \pm 5.1745 i$ & 0.0279 & $-0.2852 \pm 5.1659 i$ & 0.0551 \\
\hline \multirow[t]{11}{*}{ Local mode } & $0.3612 \pm 7.6769 i$ & -0.047 & $-1.5279 \pm 7.2691 i$ & 0.2057 \\
\hline & $0.1585 \pm 7.7144 i$ & -0.0205 & $-0.8053 \pm 7.7587 i$ & 0.1032 \\
\hline & $0.5756 \pm 7.8006 i$ & -0.0736 & $-0.9243 \pm 7.8664 i$ & 0.1167 \\
\hline & $-0.2393 \pm 8.3248 i$ & 0.0287 & $-1.6875 \pm 8.1482 i$ & 0.2028 \\
\hline & $0.1999 \pm 8.4619 i$ & -0.0236 & $-2.8534 \pm 8.1481 i$ & 0.3305 \\
\hline & $0.3218 \pm 8.6487 i$ & -0.0372 & $-0.5591 \pm 8.8129 i$ & 0.0633 \\
\hline & $0.0772 \pm 8.6898 i$ & -0.0089 & $-3.1837 \pm 8.3750 i$ & 0.3553 \\
\hline & $-0.3391 \pm 10.1725 i$ & 0.0333 & $-0.5674 \pm 9.3773 i$ & 0.0604 \\
\hline & $-0.1125 \pm 10.2137 i$ & 0.011 & $-3.7882 \pm 8.5973 i$ & 0.4032 \\
\hline & $-0.5583 \pm 10.2545 i$ & 0.0544 & $-0.6147 \pm 10.2740 i$ & 0.0597 \\
\hline & $0.1645 \pm 12.5962 i$ & -0.0131 & $-4.1247 \pm 9.4774 i$ & 0.3991 \\
\hline
\end{tabular}


HVDC-WASC to the variation of operating conditions (Fig. 5).

\subsection{Nonlinear simulation}

To examine the damping performance of the optimized controllers to multiple oscillation modes, two cases of operation are presented and analyzed.

1) Case-1: Line Outage: the tie-line 1-2, one of the backbone interconnected line between Area-4 and -5, is set to be in power outage to examine the stability of the system without, with the initial or with the optimized controllers, respectively.

Figure 6 shows the dynamic responses of the power flow. It can be seen that when the initial controllers are used, the line outage excites serious power oscillations, which leads to system collapse. However, when using the optimized LPSSs and HVDC-WASC, power oscillations are effectively damped.

Moreover, dynamic responses of the relative angle between G7 (in Area-5) and G15 (in Area-2), G16 (in Area-3) and G14 (in Area-1), and G10 (in Area-4) and G1 (in Area-5) are shown in Fig. 7 respectively, as to illustrate the damping performance on the oscillations among different generators located at different areas. It can be seen that the oscillations are damped effectively when using the optimized control parameters.

Dynamic responses of HVDC-WASC output are shown in Fig. 8. As mentioned in section 2, the control output is the supplementary control input of the converter's polecontroller (constant dc-current controller) at the rectifier side of HVDC system. From Fig. 8, it can be seen that both the initial and the optimized HVDC-WASC can response to the damping on power oscillations. However, with the coordination of the LPSSs, the optimized HVDC-WASC could maintain the stable control performance.

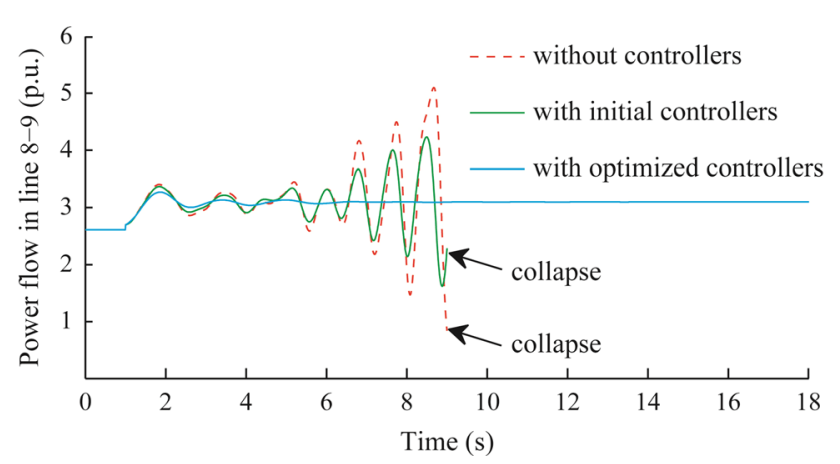

Fig. 6 Dynamic responses of the power flow in backbone tie-line 8-9 when line $1-2$ is in power outage
2) Case-2: Line Fault: In this case, the line-to-ground fault nearby bus 46 of the interconnected line 46-49 is considered to examine the stable operating ability of the system with the optimized LPSSs and HVDCWASC. Dynamic responses of the power flow in the interconnected line, relative angle between different generators, and HVDC-WASC output, are shown in Fig. 9 to Fig. 11 respectively. Fig. 9 shows that the optimized controllers can effectively damp the power oscillations in the interconnected lines. Fig. 10 shows

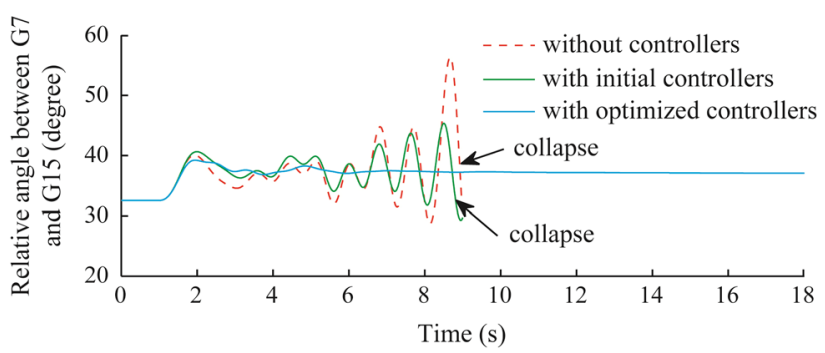

(a) Between G7 and G15

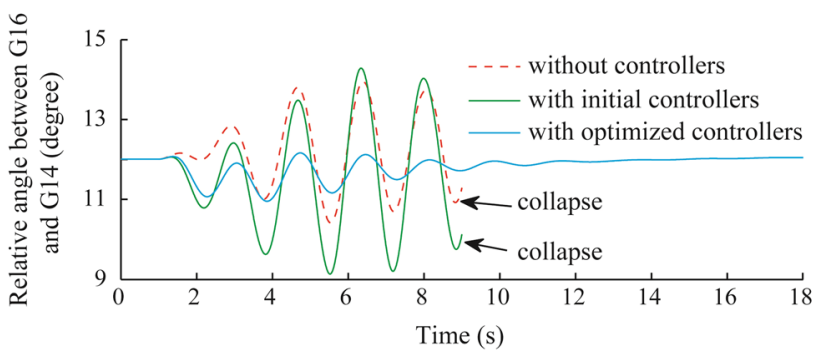

(b) Between G16 and G14

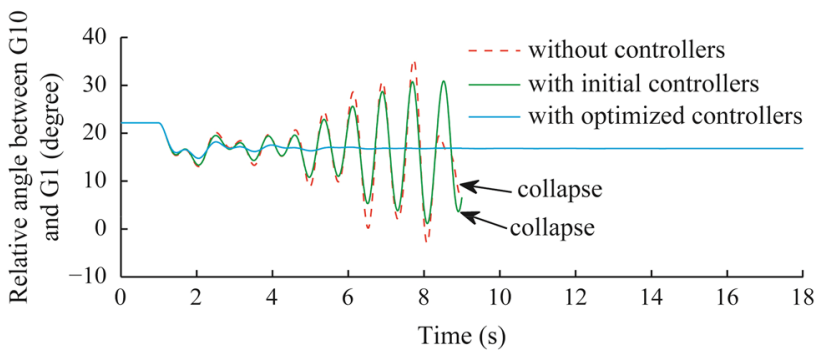

(c) Between G10 and G1

Fig. 7 Dynamic responses of relative angle between different generators when line $1-2$ is in power outage

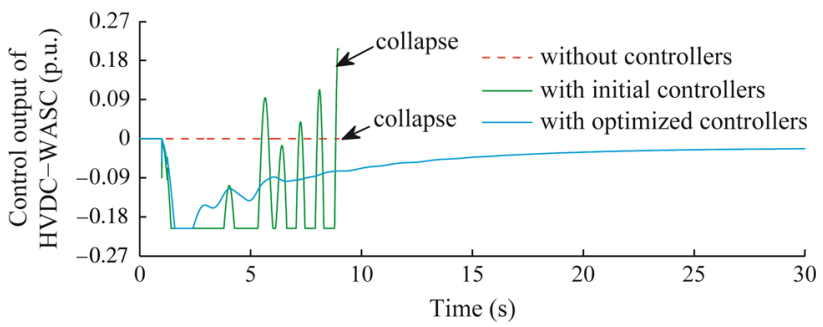

Fig. 8 Dynamic responses of the HVDC-WASC when line 1-2 is in power outage 


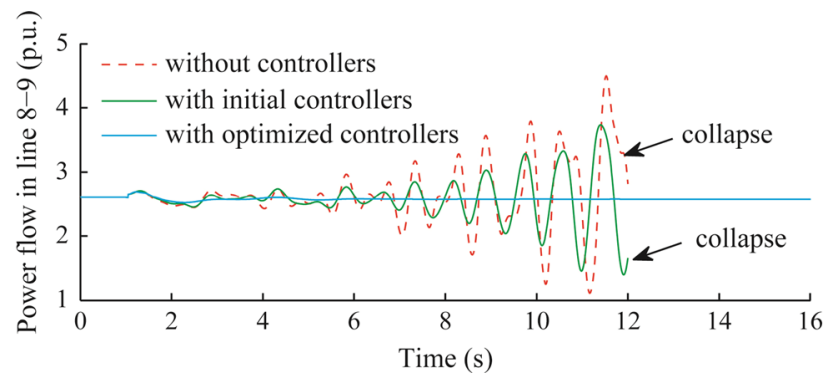

Fig. 9 Dynamic responses of the power flow in backbone tie-line 8-9 when there is line-to-ground fault nearby bus 46 of line $46-49$

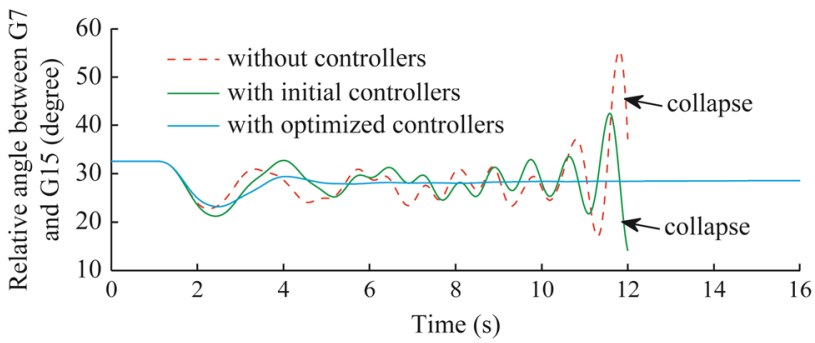

(a) Between G7 and G15

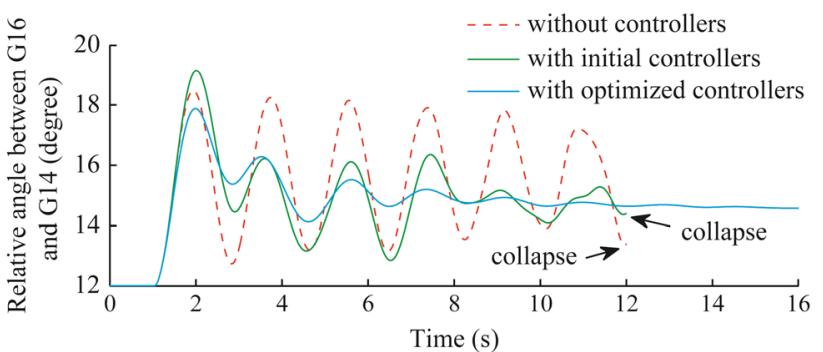

(b) Between G16 and G14

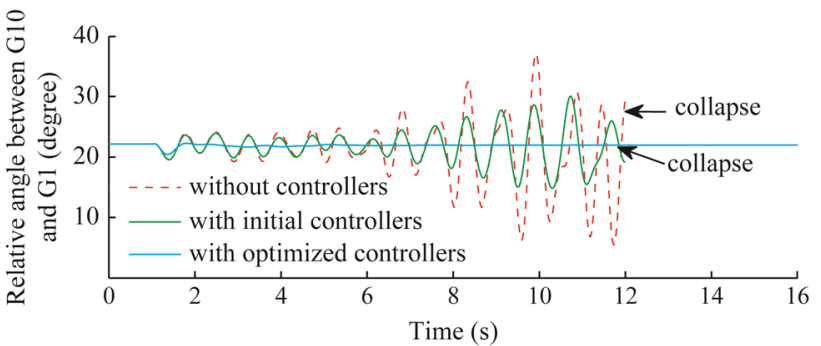

(c) Between G10 and G1

Fig. 10 Dynamic responses of relative angle between different generators when there is line-to-ground fault nearby bus 46 of line 46-49

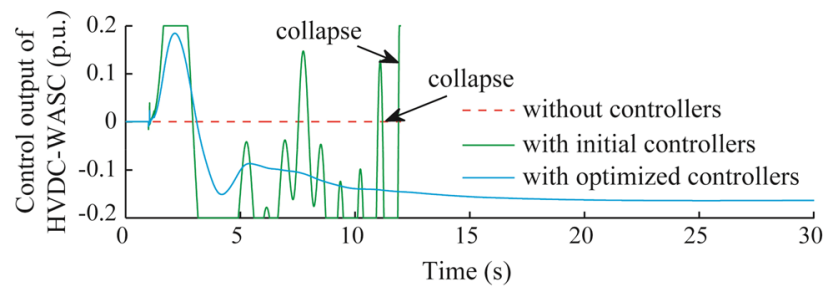

Fig. 11 Dynamic responses of the HVDC-WASC when there is line-toground fault nearby bus 46 of line 46-49 that the optimized controllers can effectively maintain the stable operation of the different generators at the different areas. Fig. 11 shows that HVDC-WASC could be in fast response to damping oscillations.

\section{Conclusion}

In this paper, a sequential design and global optimization method is proposed to simultaneously design local and wide-area stabilizing controllers, in purpose of damping local and inter-area oscillations. The guideline of sequential design is presented, and the objective function with adaptive searching ability is proposed. Further, the optimization algorithm is presented based on nonlinear optimization program. Both the eigenvalue analysis and the nonlinear simulation validate the stability of the system with the optimized LPSSs and HVDC-WASC. With the proposed method, local and wide-area controllers could be configured in global range and overall stability enhancement could be achieved, which benefits the future smart transmission grid with the advanced wide-area controls and enhanced control center functions.

Acknowledgement This work was supported by the national Natural Science Foundation of China (NSFC) (No. 51377001, No. 61233008, No. 61304092, ) the International Science and Technology Cooperation Program of China (No. 2015DFR70850), the State Grid Science and Technology Project of China (No. 5216A014007V), and the Science and Technology Project of Hunan Power Company of China (No. 5216A213509X).

Open Access This article is distributed under the terms of the Creative Commons Attribution 4.0 International License (http:// creativecommons.org/licenses/by/4.0/), which permits unrestricted use, distribution, and reproduction in any medium, provided you give appropriate credit to the original author(s) and the source, provide a link to the Creative Commons license, and indicate if changes were made.

\section{References}

[1] Bose A (2010) Smart transmission grid applications and their supporting infrastructure. IEEE Trans Smart Grid 1(1):11-19

[2] de la Ree J, Centeno V, Thorp JS et al (2010) Synchronized phasor measurement applications in power systems. IEEE Trans Smart Grid 1(1):20-27

[3] Jiang ZH, Li FX, Qiao W et al (2009) A vision of smart transmission grids. In: Proceedings of the 2009 IEEE power and energy society general meeting, Calgary, Canada, 26-30 Jul 2009, $10 \mathrm{pp}$

[4] Atanackovic D, Clapauch JH, Dwernychuk G et al (2008) First steps to wide area control. IEEE Power Energy Mag 6(1):61-68

[5] Xue YS (2008) Some viewpoints and experiences on wide area measurement systems and wide area control systems. In: Proceedings of the 2008 IEEE power and energy society general 
meeting-conversion and delivery of electrical energy in the 21st century, Pittsburgh, PA, USA, 20-24 Jul 2008, 6 pp

[6] Westermann D, Sauvain H (2009) Power system operation with wide area control including real time ATC calculation and power flow control. In: Proceedings of the 2009 IEEE Bucharest PowerTech conference, Bucharest, Romania, 28 Jun-2 Jul, 2009, 5 pp

[7] Zima M, Larsson M, Korba P et al (2005) Design aspects for wide-area monitoring and control systems. Proc IEEE 93(5):980-996

[8] Taylor CW, Erickson DC, Martin KE et al (2005) WACS-widearea stability and voltage control system: $R \& D$ and online demonstration. Proc IEEE 93(5):892-906

[9] Mao XM, Zhang Y, Guan L et al (2008) Improving power system dynamic performance using wide-area high-voltage direct current damping control. IET Gener Transm Distrib 2(2):245-251

[10] Li P, Wu XC, Lu C et al (2009) Implementation of CSG's widearea damping control system: Overview and experience. In: Proceedings of the 2009 IEEE/PES power systems conference and exposition, Seattle, WA, USA, 15-18 Mar, 2009, 9 pp

[11] Mao XM, Zhang Y, Guan L et al (2006) Coordinated control of interarea oscillation in the China Southern power grid. IEEE Trans Power Syst 21(2):845-852

[12] Do Bomfim ALB, Taranto GN, Falcao DM (2000) Simultaneous tuning of power system damping controllers using genetic algorithms. IEEE Trans Power Syst 15(1):163-169

[13] Abdel-Magid YL, Abido MA, Al-Baiyat S et al (1999) Simultaneous stabilization of multimachine power systems via genetic algorithms. IEEE Trans Power Syst 14(4):1428-1439

[14] Cai LJ, Erlich I (2005) Simultaneous coordinated tuning of PSS and FACTS damping controllers in large power systems. IEEE Trans Power Syst 20(1):294-300

[15] Kuiava R, de Oliveira RV, Ramos RA et al (2006) Simultaneous coordinated design of PSS and TCSC damping controller for power systems. In: Proceedings of the 2006 IEEE power and energy society general meeting, Montreal, Canada, 18-22 Jun 2006, 8 pp

[16] Guo C, Li QZ (2009) Simultaneous coordinated tuning of PSS and FACTS damping controller using improved particle swarm optimization. In: Proceedings of the 2009 Asia-Pacific power and energy engineering conference, Wuhan, China, 27-31 Mar $2009,4 \mathrm{pp}$

[17] Rogers G (2000) Power system oscillations. Kluwer Academic, Boston
[18] Kundur P (1994) Power system stability and control. McGrawHill, New York

Yong LI received the B.Sc. and Ph.D. degrees in 2004 and 2011, respectively, from the College of Electrical and Information Engineering, Hunan University, Changsha, China, where he is an Associate Professor of electrical engineering. Since 2009 he was also working toward the Ph.D. degree at the Institute of Energy Systems, Energy Efficiency and Energy Economics (ie3), TU Dortmund University, Dortmund, Germany and received the Ph.D. degree in 2012. His current research interests include the ac/dc energy conversion systems and equipment, analysis and control of power quality, and HVDC and FACTS technologies.

Fang LIU received the B.S. degree from the College of Electrical and Information Engineering, Zhengzhou University of Light Industry, Zhengzhou, China in 2005 and received the M.S. degree from the School of Information Science and Engineering, Central South University, Changsha, China in 2007. In 2008, she became a Ph.D. student in Waseda University, Japan and received the Ph.D. degree in 2011. She is an Associate Professor at Central South University, Changsha, China. Her main research interests include advanced control of power electronics, stability analysis of time-delay system and power systems, and robust control of FACTS with wide-area signals.

Yijia CAO graduated from Xi' an Jiaotong University, Xi' an, China in 1988 and received M.Sc. degree from Huazhong University of Science and Technology (HUST), Wuhan, China in 1991 and Ph.D. from HUST in 1994. From September 1994 to April 2000, he worked as a visiting research fellow, research fellow at Loughborough University, Liverpool University and University of the West England, UK. From 2000 to 2001, he was employed as a full professor of HUST, and from 2001 to 2008, he was employed as a full professor of Zhejiang University, China. He was appointed deputy dean of college of Electrical Engineering, Zhejiang University in 2005. Currently, he is a full professor and vice president of Hunan University, Changsha, China. His research interests are power system stability control and the application of intelligent systems in power systems. 